\title{
Analytic thermal modeling for dc-to-midrange modulation frequency responses of thin-film high- $T_{c}$ superconductive edge-transition bolometers
}

\author{
Mehdi Fardmanesh
}

\begin{abstract}
Thin-film superconductive edge-transition bolometers are modeled with a one-dimensional analytic thermal model with joule heating, film and substrate materials, and the physical interface effects taken into consideration. The results from the model agree well with the experimental results of samples made of large-meander-line $\mathrm{Yba}_{2} \mathrm{Cu}_{3} \mathrm{O}_{7-x}$ films on crystalline $\mathrm{SrTiO}_{3}, \mathrm{LaAlO}_{3}$, and $\mathrm{MgO}$ substrates up to $100 \mathrm{kHz}$, the limits of the experimental setup. Compared with the results of the $\mathrm{SrTiO}_{3}$ substrate samples, the results from the model of the $\mathrm{LaAlO}_{3}$ and the $\mathrm{MgO}$ substrate samples deviate slightly from the measured values at very low modulation frequencies (below $\sim 10 \mathrm{~Hz}$ ). The deviation increases for higher thermal-conductive substrate materials. When the model was used, the substrate absorption and the thermal parameters of the devices could also be investigated. (C) 2001 Optical Society of America

OCIS codes: $230.0040,040.3060$.
\end{abstract}

\section{Introduction}

Responsivity versus modulation frequency and versus the temperature of edge-transition superconductive bolometers has previously been investigated and reported in different studies. ${ }^{1-7}$ Major effects of the substrate-holder and the substrate-film thermal boundary resistances on the response of this kind of bolometer as well as the modulation frequency dependence of the response were reported in previous studies. ${ }^{1-7}$ From the previously reported observations, there have been some efforts to predict the response behavior by use of different thermal models for this type of detector. ${ }^{1,3,8-11}$ These efforts were in particular for the sample types used in this study. In this paper a one-dimensional thermal model and its closed-form solution are proposed for the bolometric response of thin-film high- $T_{c}$ superconductive edge-transition bolometers on thick crystalline substrates. The proposed model is for dc-to-midrange modulation frequencies above which the limits of the

M. Fardmanesh (fardman@ee.bilkent.edu.tr) is with the Electrical and Electronics Engineering Department, Bilkent University, Ankara 06533, Turkey.

Received 12 June 2000; revised manuscript received 21 August 2000.

0003-6935/01/071080-09\$15.00/0

(C) 2001 Optical Society of America heat diffusion into the film should also be further considered. The samples considered in this paper carry large-area superconductive patterns compared with the thickness of the substrates.

For the samples with large-area superconducting film patterns compared with the substrate thickness, the thermal boundary resistance at the substrateholder of the samples, $R_{s c}$, was found to be considerably higher than that of the other sources such as the film-substrate boundary resistance $R_{f}$, the substrate material resistance $R_{s}$, and the bulk film thermal resistance $R_{f}{ }^{1,3,12-14}$ Hence the total thermal conductance of the device, $R_{t}$, was close to the value of $R_{s c}$, resulting in $R_{t} \approx R_{s c}$. This is found to be the case at the low-end modulation frequencies. ${ }^{3,12}$ In such a configuration the response of the samples is expected to follow a basic two-lumped-element $R-C$ model. Then $R$ is determined mainly by the thermal boundary resistance at the substrate-holder interface (i.e., $R R_{s c}$ ), and $C$ is mainly due to the heat capacity of the substrate materials, $C_{s}$, which is much higher than that of the film for the considered samples in this paper (i.e., $C \approx C_{s} \gg C_{f}$ ). ${ }^{15-19}$ This is reported to be the case up to frequencies at which the thermal diffusion length $L$ into the substrate materials is more than the substrate thickness $L_{s}$ when the effect of the substrate-cold-finger interface can be seen by the heat wave propagating into the substrate. $^{3,12}$ The increase of the modulation frequency 
Table 1. Dimensions and Thermal Parameters of the Samples Obtained the Proposed Thermal Model ${ }^{a}$

\begin{tabular}{|c|c|c|c|c|c|c|c|c|c|c|c|}
\hline $\begin{array}{l}\text { Sample } \\
\text { Number }\end{array}$ & $\begin{array}{c}\text { Substrate } \\
\text { Material }\end{array}$ & $\begin{array}{l}d^{s} \\
(\mathrm{~cm})\end{array}$ & $\begin{array}{c}C_{s} \\
\left(\frac{\mathrm{J}}{\mathrm{k} \mathrm{cm}^{3}}\right)\end{array}$ & $\left.\begin{array}{c}K_{s} \\
\left(\frac{\mathrm{W}}{\mathrm{k} \mathrm{cm}}\right.\end{array}\right)$ & $\begin{array}{c}R_{s c} \\
\left(\frac{\mathrm{K}}{\mathrm{W} \mathrm{c \textrm {cm } ^ { 2 }}}\right)\end{array}$ & $\alpha$ & $\begin{array}{c}d^{f} \\
(\mathrm{~nm})\end{array}$ & $\begin{array}{c}A \\
\left(\mathrm{~cm}^{2}\right)\end{array}$ & $\left(\begin{array}{c}R_{f s} \\
\frac{\mathrm{mK}}{\mathrm{W} \mathrm{cm}^{2}}\end{array}\right)$ & $\left.\begin{array}{c}C_{f} \\
\left(\frac{\mu \mathrm{J}}{\mathrm{k} \mathrm{cm}^{2}}\right.\end{array}\right)$ & $\begin{array}{c}\mathrm{d} R / \mathrm{d} T \\
\left(\frac{\Omega}{\mathrm{K}}\right)\end{array}$ \\
\hline 064-03a & $\mathrm{MgO}$ & 0.05 & 0.53 & 3 & 6.82 & 2 & 170 & 0.075 & 4.4 & 642 & 1000 \\
\hline 064-01a & $\mathrm{SrTiO}_{3}$ & 0.05 & 0.43 & 0.052 & 5.6 & 1.7 & 220 & 0.017 & 44 & 655 & 1800 \\
\hline $061-02 a$ & $\mathrm{LaAlO}_{3}$ & 0.05 & 0.59 & 0.16 & 8.82 & 1 & 550 & 0.075 & 27.5 & 131 & 670 \\
\hline
\end{tabular}

${ }^{a}$ The dimensions of the substrates' area are approximately $0.5 \mathrm{~cm} \times 1 \mathrm{~cm}$ for all the samples. $\quad d_{s}$ is the thickness of the substrate, $d_{f}$ is the thickness of the YBCO film, and $R_{s c}$ is the measured substrate-cold-finger thermal boundary resistance. The factor $\alpha$ is the fitting parameter for the one-dimensional solution obtained from the model. $R_{f s}$ is the film-substrate boundary resistance, and $C_{f}$ is the heat capacity of the superconducting film obtained from the model. $A$ is the total area enclosed by the superconducting meander-line pattern.

beyond the values at which $L<L_{s}$ can cause a change in the slope of the response magnitude versus modulation frequency compared with that of the basic $R-C$ model. This was found to happen at a so-called knee frequency $f_{L}$, which is dependent on the substrate material and its thickness. ${ }^{1,3,12}$ Considering the thermal diffusion length as the characteristic penetration depth of the temperature variation into the substrate, we can determine the knee frequency from ${ }^{3,12}$

$$
f_{L}=\frac{D}{\pi L_{s}^{2}},
$$

where $L_{s}$ is the substrate thickness, $D=K_{s} / C_{s}$ is the thermal diffusivity of the substrate material, and $K_{s}$ and $C_{s}$ are the thermal conductivity and the specific heat (per unit volume) of the substrate materials, respectively.

A different regime of frequency response behavior has been identified based on the above conditions..$^{1,3}$ For frequencies less than $f_{L}$, the response was found to scale as $f^{-1}$ following the response for a basic $R-C$ model.3,12,15,16 For frequencies higher than $f_{L}$, the response was found to scale as $f^{-1 / 2}$ because of the variation of the effective penetration depth of the temperature variation into the substrate. ${ }^{1,3,12}$ The response follows the $f^{-1 / 2}$ dependence up to the frequencies at which the thermal resistance of the superconducting film-substrate boundary $R_{f s}$ is negligible compared with the frequency-dependent thermal conductance of the substrate materials $R_{s}$. The frequency dependence of the thermal conductance of the substrate $R_{s}$ can be determined from the thermal diffusion length as

$$
R_{s}=\frac{1}{A \beta \sqrt{C_{s} K_{s} \pi f}}
$$

where $A$ is the effective area of the conductive path, $f$ is the modulation frequency in hertz, and $\beta$ is a correction factor for the above approximation of the effective length of the ac heat flow. ${ }^{3,14}$ The effects of the temperature dependence of the thermal boundary resistance at the substrate interfaces were also found to be partly responsible for the variation of the response versus temperature while still following the basic model at any stable state. ${ }^{14}$ Hence the thermal parameters used in the equations should be adjusted for each bias temperature.
There have been different efforts made for response modeling of the edge-transition superconducting bolometers with and without consideration of the effects of thermal boundary resistance at the substrate interfaces and the self-heating or joule heating in the superconducting film.1,8,10,11 Based on the above proposed effects of the introduced parameters, successful analyses and predictions have been made of the behavior of the response versus modulation frequency curves of the samples that predict the frequencies at which the slope of the curve changes (the knee frequency). ${ }^{1,3}$ However, they have not predicted and matched the responses in all the measured modulation frequency regimes when an analytic model was used. The present proposed models have predicted and matched the measured responses of the samples at low frequencies, showing different considerable discrepancies at midrange or higher modulation frequencies. ${ }^{1,3}$ To my knowledge, in all the proposed models and solutions, only the magnitude of the response has been considered and compared with the experimental values. This is because the phase of the response was found to be a more sensitive characteristic of the response with respect to the model and the chosen or obtained thermal parameters of the device. ${ }^{14}$ In this paper the results obtained from the derived closed-form solution of the proposed one-dimensional model are presented. The results from the model are compared with both the measured values of the magnitude and the phase of the response of the samples.

\section{Samples and the Characterization Setup Configurations}

The samples studied in this paper were made of 120-550-nm-thick granular superconducting $\mathrm{YBa}_{2} \mathrm{Cu}_{3} \mathrm{O}_{7-x}$ (YBCO) films on 0.025 - to $0.05-\mathrm{cm}$ thick crystalline $\mathrm{SrTiO}_{3}, \mathrm{LaAlO}_{3}$, and $\mathrm{MgO}$ substrates. The films were deposited by off-axis planar magnetron sputtering at $\sim 720^{\circ} \mathrm{C}$ and patterned by a standard photolithography technique (positive photoresist) modified for less damage to the YBCO material. ${ }^{3}$ The patterns were made of either a $50-\mu \mathrm{m}$-wide and 1.9-cm-long meander line, giving a total area of $0.017 \mathrm{~cm}^{2}$, or a $120-\mu \mathrm{m}$-wide and 4.8-cm-long meander line, giving a total area of $0.075 \mathrm{~cm}^{2}$, as listed in Table 1 . The resistance versus temperature and the critical temperature $T_{c}$ of 


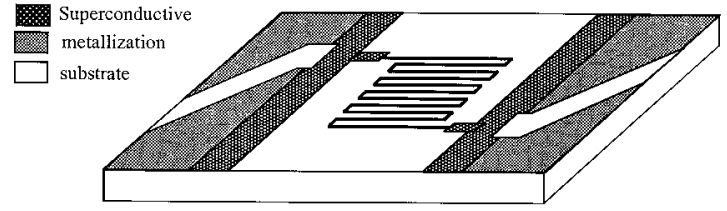

Fig. 1. Typical used superconducting thin film and the electrical contact-area patterns (metallization) of the samples on $1 \mathrm{~cm} \times 0.5$ $\mathrm{cm}$ area crystalline substrates.

the samples were found to change when the film was exposed to high bias currents or thermal cycling under vacuum, and hence the optimal bias temperature was changed. ${ }^{13}$ The transition temperatures of the samples shifted to lower temperatures because of high bias currents under vacuum and were recovered under a few thermal cycles, reaching a stable value under low bias currents. ${ }^{13}$ Hence the presented data in this paper are obtained with relatively low bias currents under stable conditions and $T_{c}$. More details about the fabrication of the samples and their dc characteristics are presented elsewhere..$^{3,13,14}$

The typical configuration of the studied samples is shown in Fig. 1. The contact areas for the four-probe measurement were coated with either an $\sim 85-\mathrm{nm}$ layer of sputtered gold or an $\sim 60$-nm layer of sputtered silver. Electrical contacts to the samples were made with copper wire (32-gauge) and silver epoxy dried at room temperature overnight. To characterize the samples, the second cold stage of a Cryo-Torr 100 was used as the cold finger by a computercontrolled heater. The temperature of the cold finger could be controlled by approximately $\pm 0.1 \mathrm{~K}$ from the set points. More details of the characterization setup and the instrumentation are presented elsewhere. ${ }^{14,17,20}$ A sample holder was designed and made of high purity and highly conductive oxygenfree $(\mathrm{OFE})$ copper that was etched in an inert environment gas and coated by a thin layer of gold without being exposed to the atmosphere. The configuration of the holder is shown in Fig. 2.

As shown in Figs. 1 and 2, there are two thermal boundary resistances at the substrate interfaces, one at the film interface and the other at the holder interface. There are two major bulk areas that con-

Superconducting Film

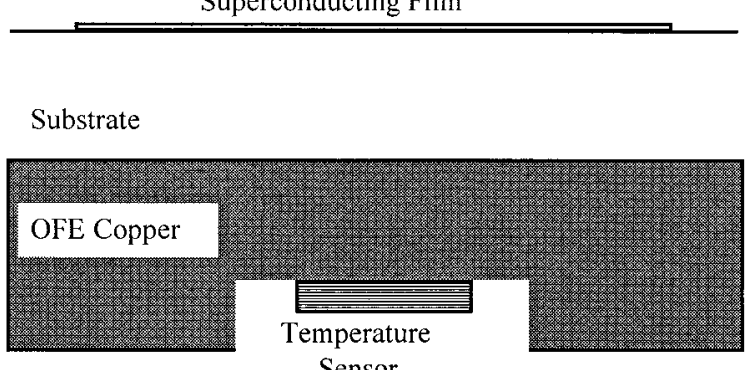

Fig. 2. Configuration of the thin superconducting film samples on a crystalline substrate in contact with the holder and the temperature sensor. tribute to the overall heat capacity of the samples, one that is due to the superconducting film and one that is due to the substrate materials. Here the OFE copper is considered to be a heat reservoir, the temperature of which is considered uniform at the value measured by the temperature sensor. To obtain a better thermal contact, a very thin layer of either Apiezon-N grease or silicon vacuum grease was used to attach the samples to the holder in the model. The effect of the grease is considered to be included in the measured substrate-holder thermal boundary resistance $R_{s c}$. The dimensional parameters and the dc electrical and thermal characteristics of interest for the studied samples in this paper are given in Table 1 . The given thermal conductivity and the heat capacity of the $\mathrm{LaAlO}_{3}$ substrate material in Table 1 are obtained by fit of the calculated response versus frequency curve from the model to that obtained experimentally.

The phase and the magnitude of the response versus modulation frequency of the samples, which are the focus of this work, were measured by a lock-in amplifier (EG\&G 5406), the input signal of which was amplified by an ultra-low-noise preamplifier (Model 030B, Perry Amplifier). A light-emitting diode (HFE4020, Honeywell) with a peak radiation wavelength of $\sim 0.85 \mu \mathrm{m}$ was used as the radiation source for the response versus modulation frequency studies in all the measurements. The diode was placed a few centimeters above the sample, providing uniform radiation on the superconducting film pattern. The response of the samples was found to be proportional to the radiation intensity up to $2.13 \mathrm{~mW} / \mathrm{cm}^{2}$, which was the limit of the source and the radiation intensity for the measurements of the results presented in this paper.

\section{Thermal Modeling}

From the dimensions given in Table 1 and also as relatively shown in Fig. 2, the area of the superconducting pattern of the samples is large with respect to the substrate thickness. Hence a one-dimensional model is considered and applied for the studied samples in this paper, with all the parameters considered for per unit area. Because of the partial lateral heat diffusion of the heat wave coming from the film into the substrate (the spreading-resistance effect), a correction factor $\alpha$ was obtained for applying to the measured $R_{s c}$ values and is given in Table 1 . The value of $\alpha$ was found to increase with the increase of the thermal conductivity of the substrate material and was highest for $\mathrm{MgO}$. It was also found to increase with a decrease in the superconducting pattern area with respect to the substrate thickness, as given in Table 1, and to be higher for the small pattern film on $\mathrm{SrTiO}_{3}$ compared with that of the $\mathrm{LaAlO}_{3}$ substrate sample. As given in Table 1, the thicknesses of the superconducting films of the samples were also very small compared with those of the substrate. This allowed the consideration of a lumped-film assumption on the top surface of the substrate in the model.

When the superconducting pattern is considered a 


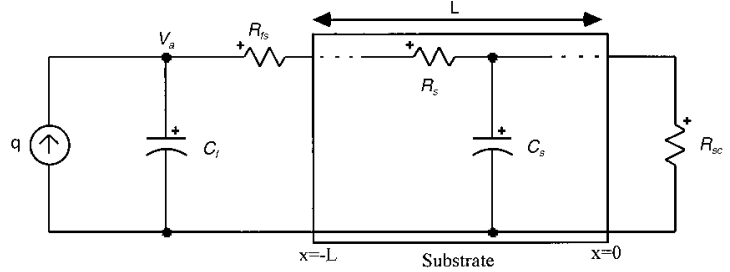

Fig. 3. Equivalent thermophysical diagram of the samples in contact with the cryogenic holder, where $q$ is the input radiation power, $C_{f}$ is the lumped heat capacitance of the film, $C_{s}$ and $R_{s}$ are the heat capacity and the thermal conductivity of the substrate material, respectively, $R_{f s}$ and $R_{s c}$ are the thermal boundary resistance values at the film-substrate and the substrate-holder interfaces, respectively, and $V_{a}$ is the temperature of the film.

lumped film and the substrate a continuous uniform medium, the thermal equivalent circuit of the samples in contact with the holder can be represented as shown in Fig. 3, where $q$ is the absorbed radiation intensity at the surface of the sample. In this equivalent diagram, two thermal boundary resistances are considered at the substrate interfaces. The heat capacitance of the superconducting film is also considered to be a value representing the total heat capacity of the film per unit area. The thermal conductance of the superconducting film was calculated and found to be negligible compared with that of the substrate and the interfaces. For samples with thicker superconducting films, as for the screenprinted materials, ${ }^{17}$ the limited heat conductivity of the superconducting material, which is the same as that for the substrate, should also be considered. Based on the above considerations in the model as shown in Fig. 3, the derivation of the temperature variation and the corresponding thermal parameters for the samples under a constant bias current are presented in the following subsection.

\section{A. Temperature Variation in the Substrate}

To approach the complete closed-form solution to the model given in Fig. 3, first we consider the absorbed radiation power $q$ to be applied directly to the surface of the substrate. This is ignoring the effects of the surface boundary conditions of $C_{f}$ and $R_{f s}$, which represent the effect of the thermal parameters of the superconducting film at this stage. This particular assumption and the solution to it would be the case for low modulation frequencies. At low modulation frequencies, $C_{f}$ is much smaller than the contributing heat capacity of the substrate, which can be determined from Eq. (1). ${ }^{3}$ Also, the film-substrate thermal boundary resistance $R_{f s}$, is much smaller than that of the substrate-holder or the low-frequency thermal resistance of the substrate $R_{s}$, determined from Eq. (2).

Based on the above assumptions and the steadystate solution to a general one-dimensional heat propagation equation, the temperature variation at the surface of the substrate, $\Delta T$, is obtained. Without consideration of the effect of the superconducting film (i.e., $C_{f}$ and $R_{f s}$ ), $\Delta T$ is obtained as follows:

$$
\Delta T=q \frac{\exp (\gamma L)+\Gamma \exp (-\gamma L)}{\exp (\gamma L)-\Gamma \exp (-\gamma L)}\left(\frac{1}{j \omega c_{s} k_{s}}\right)^{1 / 2},
$$

where $\gamma$ is the characteristic thermal impedance of the substrate material defined as

$$
\begin{gathered}
\gamma=\frac{1+j}{(2)^{1 / 2}}\left(\frac{\omega c_{s}}{k_{s}}\right)^{1 / 2}, \\
\Gamma=\frac{R_{s c}-\left(\frac{1}{j \omega c_{s} k_{s}}\right)^{1 / 2}}{R_{s c}+\left(\frac{1}{j \omega c_{s} k_{s}}\right)^{1 / 2}}
\end{gathered}
$$

where $\omega$ is the angular modulation frequency and $k_{s}$ and $c_{s}$ are the heat conductivity and the heat capacity of the substrate materials, respectively. The value of $R_{s c}$ can be found from

$$
R_{s c}=\frac{1}{G(W / K) / A},
$$

where $G$ is in units of watts per degrees kelvin and is the total thermal conductance of the device at dc or low-end modulation frequencies $G(0)$, and $A$ is the film pattern area. This is based on the assumption that $G_{f s} \gg G_{s} \gg G_{s c}$, where $G_{f_{s}}=1 / R_{f s}, G_{s}$ is the total heat conductance of the substrate, and $G_{s c}=1 / R_{s c}$. This assumption has been verified for samples with dimensions close to those of our samples, which is discussed in detail and presented elsewhere. .,3,12,14,21,22 $^{2}$

\section{B. Temperature Variation at the Film}

To find the voltage response to the modulated radiation intensities, the temperature variation in the superconductive film of the samples was obtained for all considered frequency ranges. This temperature variation corresponds to the temperature change across $C_{f}$ in the equivalent circuit of Fig. 3. Hence the only temperature gradient from the top of the superconducting film into the substrate would be that across the film-substrate interface $R_{f_{s}}$. When the one-dimensional thermal differential equation is solved with complete boundary conditions, as shown in Fig. 3 (i.e., with consideration of $C_{f}$ and $R_{f s}$ ),

$$
\Delta T=q\left[\frac{\frac{\exp (\gamma L)+\Gamma \exp (-\gamma L)}{\exp (\gamma L)-\Gamma \exp (-\gamma L)}\left(\frac{1}{j \omega c_{s} k_{s}}\right)^{1 / 2}+R_{f s}}{\frac{\exp (\gamma L)+\Gamma \exp (-\gamma L)}{\exp (\gamma L)-\Gamma \exp (-\gamma L)}\left(\frac{j \omega}{c_{s} k_{s}}\right)^{1 / 2} C_{f}+1+j \omega C_{f} R_{f s}}\right],
$$


the temperature variation caused by the radiation absorption in the film is found as follows:

where $q$ is the absorbed radiation power in the film per unit area, and $C_{f}$ and $R_{f s}$ are the total heat capacity of the superconducting film and the thermal boundary resistance at the film-substrate interface per unit area, respectively.

The obtained solution of Eq. (7) is up to frequencies at which the thermal diffusion length into the superconducting film (for YBCO material) becomes smaller or comparable with the thickness of the film. This occurs while the penetration depth or the absorption coefficient length in the superconducting film is still considered to be much smaller than the film thickness. This frequency regime for the samples of Table 1 with a maximum film thickness of $\sim 550 \mathrm{~nm}$ is found to be much higher than that investigated in this study. As given in Eq. (7), at low frequencies the denominator will approach 1 and $R_{f s}$ will become negligible compared with the first term of the numerator, leading to the results of the one boundary condition problem, as given in Eq. (3). This is because at low modulation frequencies (below $\sim 10 \mathrm{~Hz}$ in these samples) the temperature variation (heat wave) reaches the bottom of the substrate and hence the heat capacity of the film with respect to that of the whole substrate material under the pattern, and also the thermal boundary resistance at the filmsubstrate with respect to $R_{s}$ and $R_{s c}$ becomes negligible. ${ }^{3,12}$

\section{Voltage Response and Bias Current Dependence}

Considering a uniform temperature variation in the film, caused by the absorbed radiation $\Delta T$, we can obtain the voltage response in a dc bias configuration from

$$
\mathrm{d} V=I_{b} \Delta T \frac{\mathrm{d} R}{\mathrm{~d} T},
$$

where $I_{b}$ is the dc bias current, $\mathrm{d} R / \mathrm{d} T$ is the slope of the $R$ versus $T$ curve at the bias temperature as given in Table 1 , and $\Delta T$ is the temperature variation in the superconducting film as given in Eq. (7). Equation (8) is valid within the temperature variation range within which $\mathrm{d} R / \mathrm{d} T$ can be considered constant. The maximum temperature variations of the films caused by the radiation were typically below approximately a few millidegrees kelvin, as measured during the characterizations presented in this paper. The transition widths of the samples were typically a few degrees kelvin, and hence the variation of $\mathrm{d} R / \mathrm{d} T$ or the nonlinearity of Eq. (8) was found to be negligible in the measured and the calculated responses. However, this would not be the case for devices exposed to high radiation intensities that are normally voltage biased for further stability. ${ }^{23,24}$ The effect of the bias configuration on the response and the feedback effect of the joule heating for each case of the voltage-biased and current-biased configurations are discussed and presented elsewhere. ${ }^{13}$

There is also a consecutive temperature variation that is due to ac joule heating in the film caused by the resistance variation in the superconducting film that is due to the input radiation power. This effect on the response is in the form of positive feedback in the used current-biased configuration as used for the samples in this work. This ac joule heating in a first-order approximation can be considered either as a dependent radiation (or heating) source in parallel to the external radiation source $q$, as shown in Fig. 3,25 or simply as an additional term in the overall responsivity of the sample as given in the following form $^{3,12}$

$$
r_{v-t}=\frac{r_{v}}{\left(1-I r_{v} / \eta\right)},
$$

where $r_{v-t}$ is the overall responsivity in volts per watt and $\eta$ is the absorption coefficient in the film. When the effect of the absorption coefficient in the $q$ factor in Eq. (7) is considered, $r_{v}$ will be proportionally related to the $d V$ given in Eq. (8). The effect of ac joule heating in the response of the samples studied in this study is found to be negligible for the used biased currents.

\section{Measured Frequency Response and the Results from the Model}

The results from the model were compared with the experimental values for the samples given in Table 1. The frequency response behavior of the samples was found to be strongly dependent on the film and the substrate dimensions as well as on the thermal parameters, whereas the observed dependencies were in good agreement with the thermal model. To calculate the response by use of Eq. (7), the dc thermal parameters of $R_{f s}, R_{s c}, C_{f}, C_{s}$, and $K_{s}$ of the samples were determined. The measured values of the above parameters are given in Table 1. The values of $R_{s c}$, which are much higher than $R_{s}$ and $R_{f s}$, were measured by the method of the resistive or the dc joule heating in the film close to the transition temperature $T_{c}{ }^{14-16,18}$ In this method a high bias current is passed through the film and we measure the temperature rise that is due to the joule heating by measuring the resistance of the film. Then, knowing the temperature rise and the joule heating power, we obtain the values of $R_{s c}$. Parameters such as the film-substrate thermal boundary resistance and the thermal parameters of the substrate or the heat capacity of the samples could also be obtained and verified by use of the fit of the results from the model to the measured values. The values in Table 1 are given per unit area as used in the one-dimensional solution of the model.

The results from the model were investigated for thin-film samples on crystalline $\mathrm{SrTiO}_{3}, \mathrm{LaAlO}_{3}$, and $\mathrm{MgO}$ substrate materials. The measured and the calculated magnitude and phase of the response of the meander-line-patterned YBCO films on the 0.05cm-thick $\mathrm{SrTiO}_{3}, \mathrm{LaAlO}_{3}$, and $\mathrm{MgO}$ substrate samples are shown in Figs. 4, 5, and 6, respectively. The response calculated with the basic $R$ - $C$ model used in 


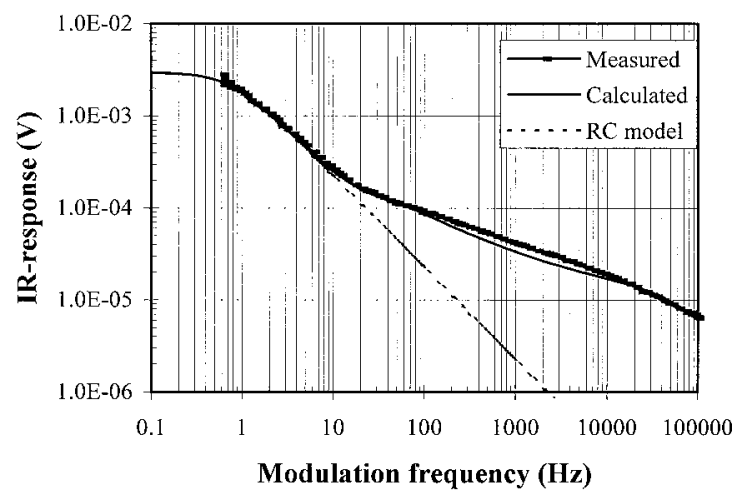

(a)

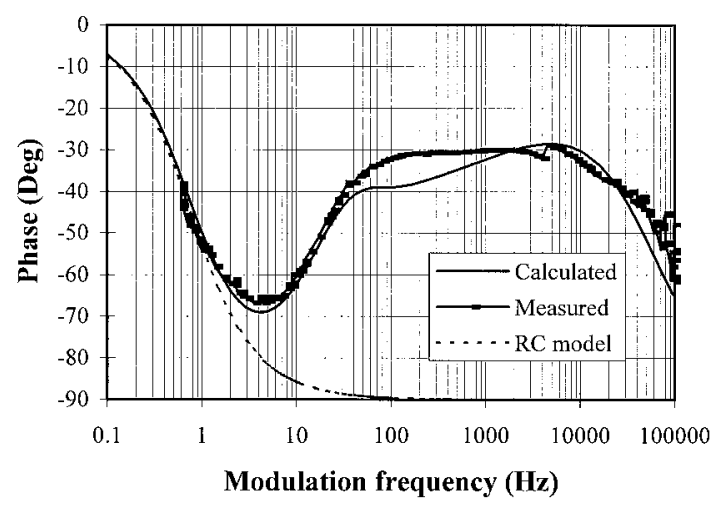

(b)

Fig. 4. Response versus modulation frequency of the $\mathrm{SrTiO}_{3}$ substrate sample 064-01a at $80 \mathrm{~K}$ and $250-\mathrm{mA}$ dc bias current. Measured and calculated (a) magnitude and (b) phase of the response from both the proposed model and the basic $R-C$ model.

Ref. 3 is also plotted for samples 064-01a and 06102Aa. The calculated response for the basic $R-C$ model is found with

$$
r_{v}=\frac{\eta I}{G+j 2 \pi f C} \frac{\mathrm{d} R}{\mathrm{~d} T},
$$

where $G$ and $C$ are the total thermal conductance and the heat capacity of the device, respectively. The basic $R-C$ model is found to be as useful as the complete model in the low-frequency ranges in which the thermal diffusion length into the substrate is much larger than the substrate thickness. This basic $R-C$ model is found to be appropriate for micromachined or freestanding film bolometers. The $G$ and the $C$ values of such bolometers would be constant up to high frequencies at which the thermal diffusion length into the substrate or into the micromachined freestanding base element of the bolometer becomes smaller or comparable with the thickness of the substrate or the base element. ${ }^{15,16}$ In general, the use of the basic $R-C$ model is appropriate in the frequency regimes in which one period of the modulation frequency is longer than the low-frequency time response of the samples, $\tau_{L}$. The $\tau_{L}$ is defined as $\tau_{L}=$ $C(0) / G(0)$, where $G(0)$ and $C(0)$ are the total low or so-called dc thermal conductance and the heat capac-

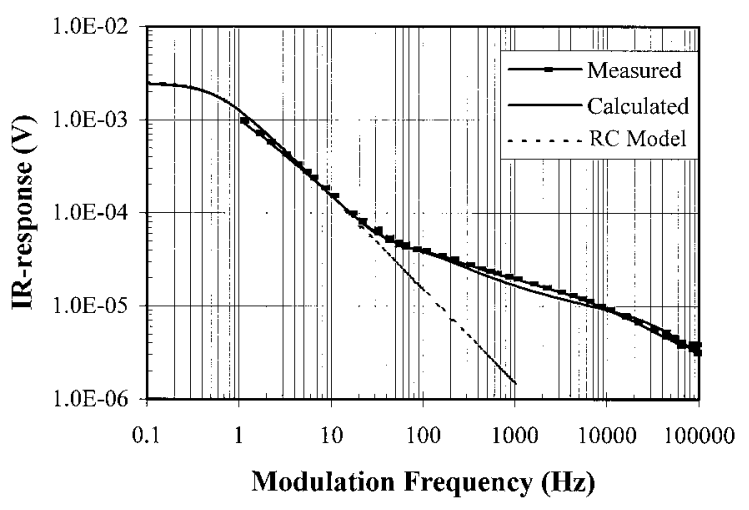

(a)

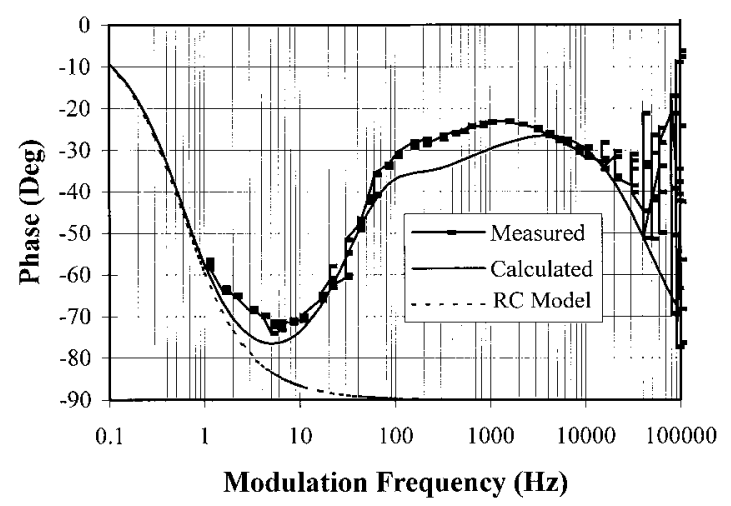

(b)

Fig. 5. Response versus modulation frequency of the $\mathrm{LaAlO}_{3}$ substrate sample 061-02a at 79.5 $\mathrm{K}$ and 500-mA bias current. Measured and calculated (a) magnitude and (b) phase of response from both the proposed model and the basic $R-C$ model.

ity of the samples, respectively. In Subsections 4.A4.C we compare the results from our model with the measured values and investigate the effects of different thermal and dimensional parameters on the fit of the model to the experimental values. The comparison is done for both the magnitude and the phase of the response in three regimes of low, middle, and high ranges of frequencies.

\section{A. Magnitude of Response at Low Frequencies and the} Dependence on the Substrate Material and Dimensions

As given in Table 1, the substrate-holder thermal boundary resistances of the samples $R_{s c}$ are given in per unit area and are found by use of the measured dc thermal conductance of the samples by the shift in the temperature caused by joule heating at elevated bias currents. ${ }^{14}$ As introduced in Section 3, the factor $\alpha$ was defined for the correction of the $R_{s c}$ value and was found to depend on the area of the pattern $A$, the substrate thickness $d_{s}$, and the thermal conductivity of the substrate materials $k_{s}$. It increases by the increase of $k_{s}$ and the increase of the ratio of $d_{s} / A$, as expected. The requirement of the factor $\alpha$ for a better fit of the calculated response curves to the measured ones is interpreted to be due to the spreading thermal resistance effect in the substrate mate- 


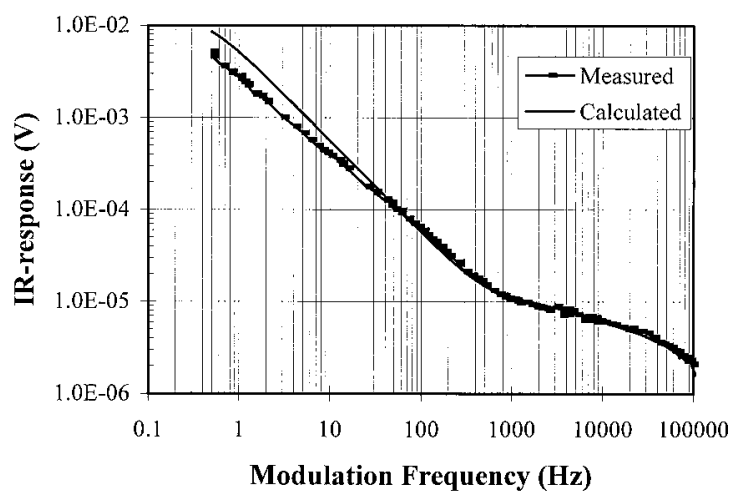

(a)

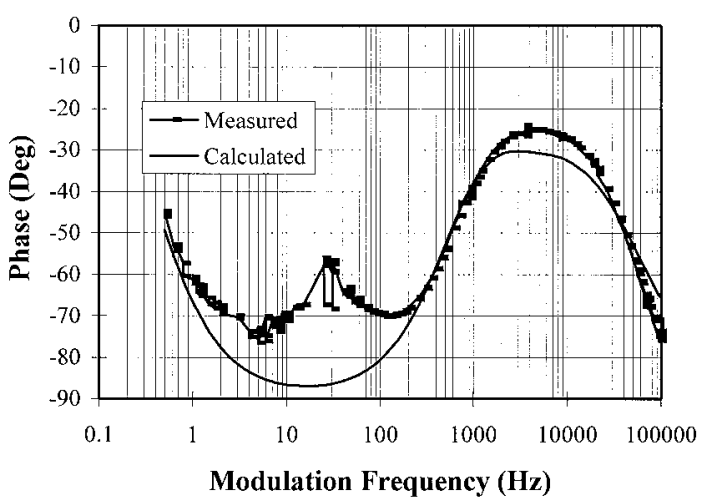

(b)

Fig. 6. Response versus modulation frequency of the 0.5 -cm-thick $\mathrm{MgO}$ substrate sample 064-03a at $79 \mathrm{~K}$ and 1-mA bias current. Measured and calculated (a) magnitude and (b) phase of the response.

rial neglected by the one dimensionality of the model. Hence, for a relatively low thermal-conductive substrate sample with a small $d_{s} / A$ ratio, this factor is expected to approach 1 as obtained for sample 06102a. By use of the known parameters for the films and the substrates and the obtained values for the $\alpha$ and the boundary resistance, the phase and the magnitude of the response for the samples were calculated and plotted in Figs. 4-6.

As shown in Figs. 4-6, at low modulation frequencies there is a discrepancy between the results from the model and the measured values. This discrepancy was found to increase by the increase of the thermal conductivity of the substrate materials, showing its maximum for the most conductive substrate material. This is also interpreted to be due to the one dimensionality of the model, ignoring the lateral heat diffusion in the substrate materials underneath the pattern. When the magnitudes of the response curves in Figs. 4, 5, and 6, for $\mathrm{SrTiO}_{3}$, $\mathrm{LaAlO}_{3}$, and $\mathrm{MgO}$, respectively, are compared, the discrepancy is the most for the most thermalconductive $\mathrm{MgO}$ substrate sample 064-03a of this work. This is also consistent with the variation of $\alpha$, which is the highest for the same sample. It is proposed that this discrepancy be avoided by use of a three-dimensional solution for the model or use of an approximate cylindrical solution for a truncated cone structure with a linearly increasing base from the film to the bottom of the substrate. Such approximate solutions can also be applied with simulation programs such as SPICE, in which the parameters can further be considered as frequency dependent. ${ }^{3}$ The preceding alternative approaches that can be applied to any kind of bolometers are presently under way and will be presented elsewhere.

\section{B. Phase of the Response and the High- and the Low-End-Frequency Behavior}

The phase of the response of the samples was found to be much more sensitive to the values of the characteristic parameters of the samples. In particular, the drop of the phase at high-end-frequency response was the determining factor in finding the equivalent values of $C_{f}$ and $R_{f s}$ given in Table 1 . Although the low-end calculated phase of the response is in good agreement with the experimental results for $\mathrm{SrTiO}_{3}$ and $\mathrm{LaAlO}_{3}$ substrate samples, there is a peak in the frequency range of $10-100 \mathrm{~Hz}$ in the $\mathrm{MgO}$ substrate sample that cannot be explained by this model. This behavior was also observed for all other $\mathrm{MgO}$ substrate samples. This was found to occur at lower frequencies in thinner substrate samples, e.g., at approximately $10-20-\mathrm{Hz}$ frequencies for $0.025-\mathrm{cm}$-thick $\mathrm{MgO}$ substrate samples compared with those of 0.5$\mathrm{cm}$-thick $\mathrm{MgO}$ substrate sample 064-03a shown in Fig. 6(b). One speculated mechanism for this is interpreted as possibly being due to the reflection and the interference effect of the acoustical phonons within the boundaries of the substrate underneath the superconducting pattern. ${ }^{14}$ The possible effect of the superconducting material on the spectral phonon density in the substrate and its consequent effects have already been observed in the phase of the response versus temperature of the samples and are presented and discussed elsewhere. ${ }^{14}$

\section{Midrange Frequency Behavior of the Phase and the Magnitude of the Response}

There is a slight discrepancy in the midrange frequency response curves compared with that of the model. This is clearly seen in the plateau of the phase versus frequency curves for all the samples shown in Figs. 4-6. The discrepancy can also be seen partly in the magnitude of the response curves in the same frequency regime. The measured response in this range was found to be slightly higher in magnitude and more in phase with the radiation signal than the expected values from the model.

The possible effect of the radiation absorption by the open areas of the substrate within the meander lines of the patterns was investigated as a possible source for the discrepancy. This consideration in the model led to a lower phase of the response increasing the observed discrepancy in the plateau region of the phase curves, particularly for the $\mathrm{SrTiO}_{3}$ substrate sample. The spectral absorption of the crystalline substrate materials was also measured 


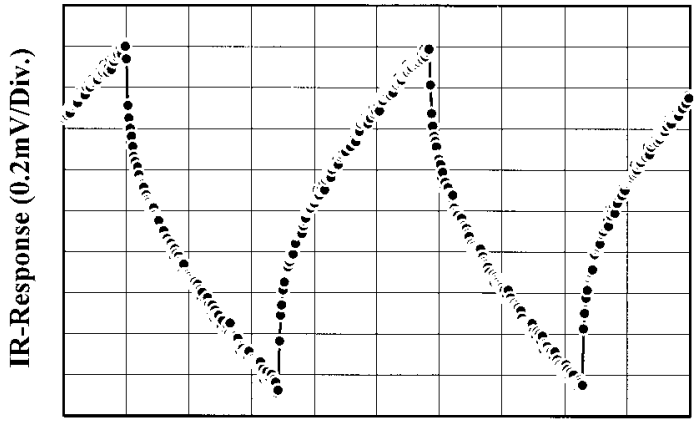

Time (10mSec./Div.)

Fig. 7. Steady-state response versus time of $\mathrm{SrTiO}_{3}$ substrate sample 064-01a at the 560-mA bias current, radiated by a $20-\mathrm{Hz}$ square radiation signal with $2.13-\mathrm{mW} / \mathrm{cm}^{2}$ maximum intensity.

from $600 \mathrm{~nm}$ to $50 \mu \mathrm{m}$ and was found to be approximately $12 \%, 20 \%$, and $8 \%$ for the 0.05 -cm-thick Sr$\mathrm{TiO}_{3}, \mathrm{LaAlO}_{3}$, and $\mathrm{MgO}$ substrates, respectively, at the $850-\mathrm{nm}$ radiation wavelength. Although the effect of the substrate absorption was found to be negligible in the response of the samples measured at the 850 -nm radiation wavelength, it was found to have a major effect on the measured spectral response of the samples at longer wavelengths. ${ }^{3,7,12,20,26}$ The effect of the substrate absorption on the spectral response of the detectors in Table 1 was studied and reported elsewhere. ${ }^{7,12}$

A small and relatively fast component in the time response of the samples was observed and found to increase relatively compared with the total response as the modulation frequency increased. This could not be explained by the thermal response as modeled in this paper. The steady-state time response of Sr$\mathrm{TiO}_{3}$ substrate sample 064-01a in the abovementioned frequency regime is shown in Fig. 7. As observed from the figure, there exist an abrupt jump and drop at the edge of the on and off switching times of the radiation source. The source is a fast near-IR source that is derived by a square-wave voltage signal, as explained in Section 2. The same type of the steady-state time response is observed for all other samples in the same frequency regime. The effect of this fast component of the response is in favor of the discrepancy (i.e., lowering the phase value and increasing the magnitude of the response from those values calculated by the model). This is interpreted as the nonthermal component of the response and is a possible source for the discrepancy, which needs to be further investigated.

\section{Summary and Conclusions}

The phase and the magnitude of response versus modulation frequency of superconducting edgetransition bolometers were shown to be strongly a function of the thermal boundary resistance at the film-substrate and the substrate-holder interfaces. A one-dimensional model based on the above facts is proposed for bolometers with superconducting film patterns larger than or comparable with the sub- strate thickness. The model, while being a useful tool for design optimizations for such devices, could also be used to monitor the thermal parameters of the detectors. The results from the model confirm the major effects of the thermal boundary resistance's dominating the effects of the substrate materials at low- and high-end modulation frequencies. The obtained thermal boundary resistance at the substrateholder of the studied samples was found to be the limiting factor at the dc or low modulation frequencies.

A good fit between both the measured magnitude and the phase of the response to that obtained from the model could be obtained for the samples from very low modulation frequencies of $0.5 \mathrm{~Hz}$ up to $\sim 100 \mathrm{kHz}$, the limits of the setup. The fit matched the best for the $\mathrm{SrTiO}_{3}$ substrate sample compared with that for the $\mathrm{MgO}$ and the $\mathrm{LaAlO}_{3}$ substrates. From the high-frequency end of the response, the superconducting film-substrate thermal boundary resistance and the heat capacity of the films could be obtained. Both the film-substrate boundary resistance and the superconducting film heat capacity of the samples in this paper were found to be larger than the previously typical reported values in the literature. From the model and from the low and the midrange frequencies of the response, the heat capacity and the thermal conductivity of the used substrate materials $\left(\mathrm{SrTiO}_{3}, \mathrm{MgO}\right.$, and $\left.\mathrm{LaAlO}_{3}\right)$ and the substrateholder thermal boundary resistance could also be obtained. The obtained thermal parameters of the substrate materials agree well with the values previously reported in the literature.

In conclusion, the proposed one-dimensional thermal model can be used to monitor the thermal parameters of the superconducting film, substrate, and the thermal boundary resistance values of an edgetransition superconductive bolometer. Also, the mechanism of heat flow caused by the absorbed radiation in the samples can be investigated by use of the results from the model in this kind of device. The response versus modulation frequency of the bolometers was found to be the most informative data in this respect. The response of the bolometers with large superconducting pattern areas compared with the substrate thickness was found to be mainly bolometric at low and midrange modulation frequencies when following the results from the proposed model. Consideration of a three-dimensional solution to the model would be required for further accurate response analysis of samples with small superconducting pattern areas compared with the substrate thickness.

\section{References}

1. P. E. Phelan, "Thermal response of thin-film high- $T_{c}$ superconductors to modulated irradiation," J. Therm. Phys. Heat Transfer 9, 397-402 (1995).

2. S. Zeuner, W. Prettl, and H. Lengfellner, "Fast thermoelectric response of normal state $\mathrm{YBa}_{2} \mathrm{Cu}_{3} \mathrm{O}_{7-d}$ films," Appl. Phys. Lett. 66, 1833-1835 (1995).

3. M. Fardmanesh, A. Rothwarf, and K. J. Scoles, "Low and 
midrange modulation frequency response for YBCO infrared detectors: interface effects on the amplitude and phase," IEEE Trans. Appl. Supercond. 5, 7-13 (1995).

4. M. Nahum, S. Verghese, and P. L. Richards, "Thermal boundary resistance for $\mathrm{YBa}_{2} \mathrm{Cu}_{3} \mathrm{O}_{7-x}$ films," Appl. Phys. Lett. 59, 2034-2036 (1991).

5. A. V. Sergeev, A. D. Semenov, P. Kouminov, V. Trifonov, I. G. Goghize, B. S. Karasik, G. N. Gol'tsman, and E. M. Gershenzon, "Transparency of a $\mathrm{YBa}_{2} \mathrm{Cu}_{3} \mathrm{O}_{7}$-film/substrate interface for thermal phonons measured by means of voltage response to radiation," Phys. Rev. B 49, 9091-9096 (1994).

6. C. D. Marshal, I. M. Fishman, R. C. Dorfman, C. B. Eom, and M. D. Fayer, "Thermal diffusion, interfacial thermal barrier, and ultrasonic propagation in $\mathrm{YBa}_{2} \mathrm{Cu}_{3} \mathrm{O}_{7-x}$ thin films: surface-selective transient-grating experiments," Phys. Rev. B 45, 10009-10021 (1992).

7. M. Fardmanesh, K. J. Scoles, and A. Rothwarf, "Control of the responsivity and the detectivity of superconductive edgetransition $\mathrm{YBa}_{2} \mathrm{Cu}_{3} \mathrm{O}_{7-x}$ bolometers through substrate properties," Appl. Opt. 38, 4735-4742 (1999).

8. K. Fushinobu, P. E. Phelan, K. Hijikata, T. Nagasaki, and M. I. Flik, "Thermal analysis of the performance of a high- $T_{c}$ superconducting bolometer," J. Heat Transfer 116, 275-279 (1994).

9. A. Frenkel, "Mechanism of nonequilibrium optical response of high-temperature superconductors," Phys. Rev. B 48, 97179725 (1993).

10. H. Neff, "Modeling and optimization of high- $T_{c}$ superconducting bolometers: the effect of film thickness," J. Appl. Phys. 69, 8375-8379 (1991).

11. M. I. Flik, P. E. Phelan, and C. L. Tien, "Thermal model for the bolometric response of high- $T_{c}$ superconducting films to optical pulses," Cryogenics 30, 1118-1128 (1990).

12. M. Fardmanesh, A. Rothwarf, and K. J. Scoles, "The responsivity and detectivity limits for patterned $\mathrm{YBa}_{2} \mathrm{Cu}_{3} \mathrm{O}_{7-x}$ superconductive IR-detectors," in Proceedings of the Sixth International Superconductive Electronics Conference, H. Kock and S. Knappe, eds. (Physikaisch-Technidsche Bundesanstalt, Berlin, 1997), Vol. 3, pp. 399-401.

13. M. Fardmanesh, K. J. Scoles, and A. Rothwarf, "DC characteristics of patterned $\mathrm{YBa}_{2} \mathrm{Cu}_{3} \mathrm{O}_{7-x}$ superconducting thin film bolometers: artifacts related to joule heating, ambient pressure, and microstructure," IEEE Trans. Appl. Supercond. 8, 69-78 (1998).

14. M. Fardmanesh, A. Rothwarf, and K. J. Scoles, "YBa $\mathrm{Cu}_{3} \mathrm{O}_{7-x}$ infrared bolometers: temperature dependent responsivity and deviations from the $d R / d T$ curve," J. Appl. Phys. 77, 4568-4575 (1995).
15. A. Jahanzeb, C. M. Travers, Z. Celik-Butler, and D. P. Butler, "A semiconductor $\mathrm{YBaCuO}$ microbolometer for room temperature IR imaging," IEEE Trans. Electron Devices 44, 17951801 (1997).

16. C. M. Travers, A. Jahanzeb, D. P. Butler, and Z. CelikButler, "Fabrication of semiconducting $\mathrm{YBaCuO}$ surfacemicromachined bolometer arrays," J. Microelectromech. Syst. 6, 271-276 (1997).

17. M. Fardmanesh, M. Ihsan, A. Rothwarf, K. Scoles, and K. Pourrezaei, "Thick and thin film Y-Ba-Cu-O infrared detectors," AIP Conf. Proc. 251, 681-691 (1991).

18. M. Nahum, Q. Hu, P. L. Richards, S. A. Sachtjen, N. Newman, and B. F. Cole, "Fabrication and measurement of high $T_{c}$ superconducting bolometers," IEEE Trans. Magn. 27, 30813084 (1991).

19. P. L. Richards, J. Clarke, R. Leoni, Ph. Lerch, S. Verghese, M. R. Beasley, T. H. Geballe, R. H. Hammond, P. Rosenthal, and S. R. Spielman, "Feasibility of the high $T$ superconducting bolometer," Appl. Phys. Lett. 54, 283-285 (1989).

20. M. Fardmanesh, A. Rothwarf, and K. J. Scoles, "Noise characteristics and detectivity of $\mathrm{YBa}_{2} \mathrm{Cu}_{3} \mathrm{O}_{7}$ superconducting bolometers: bias current, frequency, and temperature dependence," J. Appl. Phys. 79, 2006-2011 (1996).

21. H. Chou, H. Z. Chen, M. T. Hong, Y. C. Chen, and T. C. Chow, "Bolometric detection in a precipitation free $\mathrm{YBa}_{2} \mathrm{Cu}_{3} \mathrm{O}_{7-x}$ film at 77 K," Appl. Phys. Lett. 68, 2741-2743 (1996).

22. J. H. Hao, F. Q. Zhou, X. R. Zhao, H. D. Sun, X. J. Yi, and Z. G. $\mathrm{Li}$, "Responsitivity calculation and measurement of $\mathrm{YBaCuO}$ optical detector," IEEE Trans. Appl. Supercond. 3, 2167-2169 (1993).

23. G. D. Poulin, J. Lachapelle, S. H. Moffat, F. A. Hegmann, and J. S. Preston, "Current-voltage characteristics of dc voltage biased high temperature superconducting microbridges," Appl. Phys. Lett. 66, 2576-2578 (1995).

24. K. D. Irwin, G. C. Hilton, D. A. Wollman, and J. M. Martinis, "X-ray detection using a superconducting transition-edge sensor microcalorimeter with electrothermal feedback," Appl. Phys. Lett. 69, 1945-1947 (1996).

25. Z. M. Zhang and A. Frenkel, "Thermal and nonequilibrium responses of superconductors for radiation detectors," J. Supercond. 7, 871-883 (1994).

26. K. Kamaras, K. L. Barth, F. Keilmann, R. Henn, M. Reedyk, C. Thomsen, M. Cardona, J. Kircher, P. L. Richards, and J. L. Stehle, "The low-temperature infrared optical functions of Sr$\mathrm{TiO}$ determined by reflectance spectroscopy and spectroscopic ellipsometry,” J. Appl. Phys. 78, 1235-1240 (1995). 